\title{
Genomic characterization of multi-drug resistant ESBL-producing Escherichia coli ST58 causing fatal colibacillosis in critically endangered Brazilian merganser (Mergus octosetaceus)
}

\author{
Danny Fuentes-Castillo ${ }^{1,2}$, Pedro Enrique Navas-Suarez ${ }^{3}$, Maria Gondim ${ }^{4}$, Fernanda \\ Esposito $^{5}$, Carlos Sacristán ${ }^{6}$, Herrison Fontana ${ }^{2}$, Bruna Fuga ${ }^{2}$, Camila Piovani ${ }^{4}$, Robert \\ Kooij ${ }^{4}$, Nilton Lincopan ${ }^{1}$, and José Luiz Catão-Dias ${ }^{7}$ \\ ${ }^{1}$ University of São Paulo \\ ${ }^{2}$ University of Sao Paulo \\ ${ }^{3}$ Laboratório de Patologia Comparada de Animais Selvagens, Departamento de Patologia, \\ Faculdade de Medicina Veterinária e Zootecnia, Universidade de São Paulo, São Paulo, SP, \\ Brazil \\ ${ }^{4}$ Zooparque Itatiba \\ ${ }^{5}$ School of Pharmaceutical Sciences, University of São Paulo \\ ${ }^{6}$ Universidade de Sao Paulo \\ ${ }^{7}$ Faculdade de Medicina Veterinária e Zootecnia, Universidade de São Paulo
}

May 15, 2020

\begin{abstract}
Even though antimicrobial-resistant bacteria have begun to be detected in wildlife, raising important issues related to their transmission and persistence of clinically important pathogens in the environment, little is known about the role of these bacteria on wildlife health, especially on endangered species. The Brazilian merganser (Mergus octosetaceus) is one of the most threatened waterfowl in the world, classified as Critically Endangered by the International Union for Conservation of Nature. In 2019, a fatal case of sepsis was diagnosed in an 8-day-old Brazilian merganser inhabiting a zoological park. At necropsy, major gross lesions were pulmonary and hepatic congestion. Using microbiologic and genomic methods, we identified a multidrug-resistant (MDR) extended-spectrum $\beta$-lactamase (ESBL) CTX-M-8-producing Escherichia coli (designed as PMPU strain) belonging to the international clone ST58, in celomic cavity, esophagus, lungs, small intestine and cloaca samples. PMPU strain harbored a broad resistome against antibiotics (cephalosporins, tetracyclines, aminoglycosides, sulfonamides, trimethoprim, and quinolones), domestic/hospital disinfectants, and heavy metals (arsenic, mercury, lead, copper, and silver). Additionally, the virulence of E. coli PMPU strain was confirmed using a wax moth (Galleria mellonella) infection model, and it was supported by the presence of virulence genes encoding toxins, adherence factors, invasins and iron acquisition systems. Broad resistome and virulome of PMPU contributed to therapeutic failure and death of the animal. In brief, we report for the first time a fatal colibacillosis by MDR-ESBL-producing E. coli in critically endangered Brazilian merganser, highlighting that besides colonization, critical priority pathogens are threatening wildlife. E. coli ST58 clone has been previously reported in humans, food-producing animals, wildlife, and environment, supporting broad adaptation and persistence at human-animalenvironment interface.
\end{abstract}

\section{INTRODUCTION}

Antimicrobial resistance (AMR) is one of the major Global Health challenges of the 21st century, and annually kills thousands of people in the world (Cassini et al., 2019; Hernando-Amado, Coque, Baquero, 
\& Martínez, 2019). One Health and Global Health approaches are necessaries to combat the emergence, evolution and spread of AMR (Hernando-Amado et al. , 2019). In this regard, wildlife has been suggested as reservoirs, disseminators or bio-indicators of AMR in the environment (Borges et al., 2017; Dolejska \& Literak, 2019;Sacristán et al. , 2020); however, threatened wildlife species are being colonized by antibiotic resistant bacteria, but there are critical data gaps and research needs to understand the role and the real impact of AMR on wildlife (Larsson et al. , 2018; Fuentes-Castillo et al., 2020; Ramey \& Ahlstrom, 2020).

The Brazilian merganser (Mergus octosetaceus Vieillot, 1817) is one of the most threatened avian species in the Americas and one of the most threatened waterfowl in the world, classified as Critically Endangered by the International Union for Conservation of Nature (Lamas \& Lins, 2009; BirdLife International, 2019). It is estimated that its population does not exceed 250 mature individuals in nature but, thanks to conservation breeding programs, it has been possible to successfully reproduce the species ex-situ (BirdLife International, 2019).

In this study, using microbiological and whole genome sequencing tools, we investigated a fatal sepsis caused by an antibiotic-resistant bacterium in a critically endangered Brazilian merganser. In this regard, the resistome (antibiotics, heavy metals, and disinfectants), virulome, and epidemiological characteristics of the pathogen were analyzed.

\section{MATERIALS AND METHODS}

\subsection{Brazilian merganser}

As part of the Brazilian merganser Conservation Program, the Itatiba Zoological Park (Sao Paulo state, Brazil) carries out a successful breeding project. In October 2019, an 8-day-old Brazilian merganser born in the breeding program became ill presenting respiratory symptoms (dyspnea, prostration, hyporexia and weight loss). The duck received prophylactic antibiotic treatment (i.e., Enrofloxacin - Chemitril, Chemitec $15 \mathrm{mg} / \mathrm{kg}$, IM, q. 12h), with unsuccessful results. The animal died presenting incoordination and opisthotonos, less than 24 hours after the first clinical signs.

\subsection{Necropsy and sampling}

Full necropsy examination was carried out at the Laboratory of Wildlife Comparative Pathology, Department of Pathology, School of Veterinary Medicine and Animal Science of the University of São Paulo, Brazil, according to Matushima (2007). Representative samples of major organs/tissues, including esophagus, proventriculus, small and large intestines, pancreas, spleen, liver, lungs, trachea, heart, aorta, and kidney were collected and fixed in 10\% neutral buffered formalin. Central nervous system was not sampled to preserve the cranium for museum collection. Tissue samples were processed routinely and embedded in paraffin wax. Sections $(5 \mu \mathrm{m})$ were stained with hematoxylin and eosin. Additionally, selected samples from celomic cavity, oral cavity, esophagus, lungs, small intestine, and cloaca were aseptically sampled using sterilized swabs and deposited in Amies transport medium with charcoal for posterior microbiological analysis.

\subsection{Isolation, bacterial identification, and antimicrobial susceptibility testing}

Cloacal, celomic and oral cavity and tissue swab samples were streaked onto blood and MacConkey agar plates and incubated overnight at $35 \pm 2{ }^{\circ} \mathrm{C}$. Bacterial isolates were identified by the MALDI-TOFMS system (Bruker Daltonik), and clonal relationships among Escherichia coliisolates were determined by enterobacterial repetitive intergenic consensus (ERIC)-PCR (Da Silveira et al., 2002).

Antimicrobial susceptibility testing was performed by the disk diffusion method using human and veterinary antimicrobials (CLSI, 2018, 2020), including amoxicillin/clavulanate, ceftriaxone, cefotaxime, ceftiofur, ceftazidime, cefepime, cefoxitin, aztreonam, imipenem, meropenem, ertapenem, nalidixic acid, enrofloxacin, gentamicin, amikacin, trimethoprim-sulfamethoxazole and tetracycline. E. coli ATCC 25922 was used as control strain. Extended-spectrum $\beta$-lactamase (ESBL) production was screened by the double-disk synergy test (DDST) (Jarlier, Nicolas, Fournier,\& Philippon, 1988).

\subsection{Whole genome sequence (WGS) analysis}


For selected ESBL-producing E. coli strain, genomic DNA was extracted using a PureLinkTM Quick Gel Extraction Kit (Life Technologies, Carlsbad, CA), and a genomic paired-end library (75 x 2 bp) was prepared using a Nextera XT DNA Library Preparation Kit (Illumina Inc., Cambridge, UK) according to the manufacturer's instructions. The whole genome was sequenced on the NextSeq platform (Illumina). De novo genome assembly and contig annotation was carried out using CLC Genomics Workbench 12.0.3. Multilocus sequence type (MLST), plasmid replicons, resistome and serotype were identified using MLST v2.0 (Larsen et al. , 2012), PlasmidFinder v2.1 (Carattoli et al. , 2014), ResFinder v3.2 (Zankari et al. , 2012), and SerotypeFinder v2.0 (Jenkins, 2015) tools, respectively, from Center for Genomic Epidemiology (http://www.genomicepidemiology.org/). Clinically important virulence factors were detected and compared by ABRicate v0.9.8 (https://github.com/tseemann/abricate) using data from the Escherichia coli Virulence Factors (https://github.com/phac-nml/ecoli_vf) and the Virulence Factor Database (VFDB) (http://www.mgc.ac.cn/VFs/). Heavy metal (HM) and biocides genes were detected using the BacMet2 experimentally confirmed database (http://bacmet.biomedicine.gu.se). For whole genome of selected ESBLproducing $E$. coli identified in this study, a minimum spanning tree was constructed in Enterobase using the MSTree V2 algorithm and the wgMLST scheme (https://enterobase.warwick.ac.uk/species/index/ecoli). This scheme consists of 25,002 pan-genome genes present in E. coli genomes, which represented most of the diversity in Enterobase at the time (March 2020) (https://bitbucket.org/enterobase/enterobaseweb/wiki/Escherichia\%20Statistics). All images were generated with iTOL v.5.5 (https://itol.embl.de).

\subsection{In vivo virulence assays in the greater wax moth (Galleria mellonella) infection model}

In vivo virulence behavior of ESBL-producing $E$. coli was evaluated using the $G$. mellonella infection model (Tsai, Loh, \& Proft, 2016). The non-virulent E. coli ATCC 25922 and the hypervirulent meningitis/sepsis associated K1 E. coli strain (MNEC RS218) (Achtman et al., 1983; Santos, Zidko, Pignatari, \& Silva, 2013) were used as non-virulent and hypervirulent controls. In brief, G. mellonella larvae, of nearly 250 to $350 \mathrm{mg}$, were inoculated with $10^{5} \mathrm{CFU}$ of each strain. Survival of two G. mellonella groups (each group composed by 20 larvae) inoculated with each strain were evaluated for $96 \mathrm{~h}$. Data were analyzed by the log rank test, with $p<0.05$ indicating statistical significance (Prism GraphPad Software, San Diego, CA, USA).

\section{RESULTS AND DISCUSSION}

\subsection{Pathological findings}

The main gross finding was dark reddish coloration in the lungs, draining a marked amount of serosanguineous fluid. Microscopically, hemodynamic disturbances were observed in the lungs, highlighting a marked congestion of blood vessels and alveolar capillaries, and mild acute alveolar hemorrhage (Figure 1a). In liver, moderate congestion in zone I and II was detected (Figure 1b). Finally, in kidney, corticomedullar congestion was also observed. Histopathological alterations were not perceived in the remaining organs/tissues analyzed.

\subsection{Bacterial isolation, identification, and antimicrobial resistance profile}

E. coli was isolated from celomic cavity, esophagus, lungs, small intestine, and cloaca. Clonal relatedness analysis (ERIC-PCR) and antimicrobial resistance profile confirmed the infection by an identicalE. coli clone. All E. coli strains were ESBL producers and displayed a resistant profile to human and veterinary broad-spectrum cephalosporins, tetracyclines, aminoglycosides, sulfonamides, trimethoprim, and quinolones, remaining susceptible to carbapenems, cephamycin and monobactams. E. coli strain from lung tissue sample was selected to WGS analysis and designed as PMPU strain.

3.3. E. coli PMPU strain carried a wide resistome to antibiotics, heavy metals, and disinfectants

PMPU strain belonged to sequence type ST58 and serotype O102:H30. This strain harbored a resistome against antibiotics, heavy metals, and disinfectants. WGS analysis identified the presence of genes encoding resistance to cephalosporins (bla CTX-M-8 and bla TEM-1B), tetracyclines $[\operatorname{tet}(A)]$, aminoglycosides $[a p h(3 ") I b$ 
and $\operatorname{aph}(6)-I d]$, sulfonamides ( sul2 ), and trimethoprim (dfrA8). In addition, PMPU strain showed mutations in gyrA (Ser-83-Leu and Asp-87-Asn) and parC (Ser-80-Iso) genes, conferring resistance to quinolones. Moreover, genes conferring resistance to heavy metals (i.e., lead, arsenic, copper, silver, antimony, zinc, tellurium, tungsten, magnesium, cobalt, nickel, manganese, cadmium, mercury, iron, molybdenum, chromium, selenium, and vanadium), and biocides commonly used as disinfectants in domiciliary and hospital settings [i.e., quaternary ammonium compounds (QACs), acridines, chlorhexidine, sodium dodecyl sulphate, ethidium bromide, hydrochloric acid, hydrogen peroxide, and sodium hydroxide] were found (Figure 2). Regarding to plasmidome in PMPU strain, IncI1 and IncQ1 plasmid replicons were detected.

E. coli ST58 is a globally disseminated clone previously reported in humans, food-production animals, wildlife and the environment, supporting a broad adaptation, persistence and a worldwide dissemination of this clone (McKinnon, Roy Chowdhury, \& Djordjevic, 2018; Borges, Tarlton, \& Riley, 2019; Zurfluh et al., 2019; EnteroBase, 2020; De Carvalho et al., 2020). In Brazil, E. coli ST58 has been isolated from humans, poultry, peri-urban wild animals, and polluted mangrove ecosystem (Sacramento et al., 2018; Borges et al., 2019; De Carvalho et al., 2020). On the other hand, MDR or ESBL-producing E. coliserotype O102:H30 has been recurrently identified in hospitalized human patients, mainly with urinary tract infection (Gonçalves et al., 2009; Cergole-Novella et al., 2010; Cergole-Novella, Pignatari, \& Guth, 2015).

We further investigated the genomic relatedness among E. coli PMPU isolate identified in this study and 123 assembled genomes of $E$. coli belonging to ST58 from different sources of origin and countries, available in EnteroBase database (https://enterobase.warwick.ac.uk/). In the minimum spanning tree of the whole genome analysis based on the wgMLST scheme from EnteroBase, E. coli PMPU isolate showed high genetic relatedness compared to livestock isolates from Japan (ESC_QA8442AA_AS and ESC_QA8026AA_AS) and Belgium (ESC_QA7365AA_AS), an animal companion isolate from Canada (dog; ESC_YA3357AA_AS), and an environment isolate from Japan (ESC_HA7644AA_AS) (Figure 3). These phylogenetically related isolates were collected between 2013 and 2018, supporting rapid adaptation and dissemination of this $E$. coli clone.

\subsection{Virulome of ESBL-positive $E$. coli ST58 colonizing Brazilian merganser is associated with} a virulent behavior

Virulome analysis of ESBL-producing $E$. coli PMPU strain highlighted virulence factors, including adherence factors ( fim, eaeH , lpfAO113 , csgBCDEFG), invasins (iss ,ibeBC), cytolytic pore-forming toxin ( $h l y E$ ), iron acquisition systems (entBCEFS , fep $A B C D$ ) and chemotaxis (cheABRMWYZ, motAB ), among other virulence factors commonly found in commensal and pathogenic E. coli strains (Table 1). The virulent potential of PMPU strain was confirmed in the G. mellonella infection model, where strains inoculated at $1 \times 10^{5} \mathrm{CFU}$ killed $100 \%$ of wax moth larvae within $50 \mathrm{~h}$, showing a more virulent behavior than $E$. coli ATCC 25922, but no more than hypervirulent meningitis-causing E. coli MNEC RS218 (Figure S1). $G$. mellonella has been successfully utilized as anin vivo model to assess the pathogenic potential of clinically important bacterial pathogen. Therefore, responses to bacterial infections observed in this model could closely mimics responses displayed by mammalian models (Jander, Rahme, \& Ausubel, 2000; Kavanagh \& Reeves, 2004; Lange et al., 2019). In this study, virulent performance of E. coli PMPU strain was correlated with virulence factors commonly identified in pathogenic $E$. coli lineages from humans and poultry, highlighting adherence factors (fimBCEFGHI, eaeH, lpfAO113 , csgBCDEFG ) (Osek, Weiner \& Hartland, 2003; Dale \& Woodford, 2015; Torres, 2016; Sarowska et al., 2019), invasins (iss , ibeBC ) (Sarowska et al., 2019), toxin (hlyE ) (Wyborn et al., 2004), iron acquisition systems (entBCEFS , fepABCD ) (Torres, 2016; Robinson, Heffernan, \& Henderson, 2018) and chemotaxis factors (cheABRMWYZ ,motAB ) (Pettersen, Mosevoll, Lindemann, \& Wiker, 2016). In this regard, adherence factors and invasins found in the E. coli PMPU strain may have contributed to the colonization in different tissues of the bird; and, the cytolytic pore-forming toxin hlyE could be related to hemodynamic disturbances and tissue damage found in the histopathology (Oscarssonet al, 1999; Lai et al, 2000; Lithgow, Haider, Roberts, \& Green, 2007). On the other hand, the immature immune system in a 8-day-old Brazilian merganser, the artificial incubation conditions (RuizCastellano et al., 2016), as well as use of disinfectants may contributed to the selection of a virulent E. coli resistant to a wide range of antibiotics and disinfectants, establishing a disseminated infection with a fatal 
end. In order to avoid new infections due to $E$. coli widely resistant to antimicrobials and disinfectants, a cleaning of the environments was carried out using peracetic acid concentrated at $0.2 \%$. After this, no new cases of deaths occurred due to this bacterium.

Virulent pathogens resistant to an increasing number of antimicrobials cause thousands of deaths in the human population each year (Gu et al. , 2018; Cassini et al. , 2019; Centers for Disease Control, 2019). In this concern, wildlife plays an important role in the epidemiology of antibiotic-resistant pathogens in the environment (Alcalá et al. , 2016; Vittecoq et al., 2016; Sevilla et al. , 2020). However, little is known about the impact of these MDR-pathogens on wildlife, especially on threatened wildlife species (Gonçalves et al. , 2012; Larsson et al. , 2018; Ramey \& Ahlstrom, 2020). In this study, we isolated a MDR ESBL-producing $E$. coli with virulent behavior, belonging to international clone ST58 and serotype O102:H30, causing fatal infection in a critically endangered Brazilian merganser. Of note, a MDR colistin-resistantE. coli ST58 was recently isolated from a polluted mangrove ecosystem in Brazil (Sacramento et al. , 2018), being able to become a serious threat to the associated wildlife and human population.

A better integration of environmental and wildlife issues is necessary to a successful One Health approach for global AMR crisis (White \& Hughes, 2019). In this context, to understand epidemiologically the evolution and adaptation of AMR, wildlife veterinarians must increasingly report the challenges that arise when treating antimicrobial-resistant pathogenic bacteria in wildlife species. Herein, we report a fatal colibacillosis by MDRESBL-producing E. coli in critically endangered Brazilian merganser, highlighting that besides colonization, critical priority pathogens are threatening wildlife.

\section{DATA AVAILABILIT Y STATEMENT}

The whole genome nucleotide sequence of the E. coli PMPU isolate is available in the GenBank database under accession number PRJNA608189.

\section{ACKNOWLEDGEMENTS}

We thank Luís Fábio Silveira, Laura Souza, Paula Martins and Gerson Martins from Brazilian Merganser breeding project. This work was supported by Comisión Nacional de Investigación Científica y Tecnológica under Grant number CONICYT BCH 72170436; the Bill \& Melinda Gates Foundation (Grand Challenges Explorations Brazil - New approaches to characterize the global burden of antimicrobial resistance, grant OPP1193112), Conselho Nacional de Desenvolvimento Científico e Tecnológico (CNPq) under Grant number 462042/2014-6, 433128/2018-6 and 312249/2017-9 and Coordination for the Improvement of Higher Education Personnel (CAPES). J.L.C.D. is the recipient of a fellowship from CNPq under Grant number 304999-18. C.S. is a recipient of a post-doctoral fellowship by The São Paulo Research Foundation under Grant number FAPESP 2018/25069-7. N.L. is a research fellow of CNPq under Grant number 312249/2017-9.

\section{ETHICAL APPROVAL}

The authors confirm that the ethical policies of the journal, as noted on the journal's author guidelines page, have been adhered to. No ethical approval was required for this specific study.

\section{CONFLICT OF INTERESTS}

No potential conflict of interest was reported by the authors.

\section{REFERENCES}

Achtman, M., Mercer, A., Kusecek, B., Pohl, A., Heuzenroeder, M., Aaronson, W., .. Silver, R.P. (1983). Six widespread bacterial clones among Escherichia coli K1 isolates. Infection and Immunity, 39, 315-335.

Alcalá, L., Alonso, C.A., Simón, C., González-Esteban, C., Orós, J., Rezusta, A., .. Torres, C. (2016). Wild birds, frequent carriers of Extended-Spectrum $\beta$-Lactamase (ESBL) producing Escherichia coli of CTX-M and SHV-12 types. Microbial Ecology , 72, 861-869. http://dx.doi.org/10.1007/s00248-015-0718-0 
BirdLife International. (2019). Mergus octosetaceus . The IUCN Red List of Threatened Species 2019. Retrieved from https://www.iucnredlist.org/species/22680482/143756439

Borges, C. A., Beraldo, L.G., Maluta, R.P., Cardozo, M. V., Barboza, K.B., Guastalli, E.A.L., .. Ávila, F.A. (2017). Multidrug-resistant pathogenic Escherichia coli isolated from wild birds in a veterinary hospital. Avian Pathology , 46, 76-83. https://doi.org/10.1080/03079457.2016.1209298

Borges, C. A., Tarlton, N.J., \& Riley, L.W. (2019). Escherichia coli from Commercial Broiler and Backyard Chickens Share Sequence Types, Antimicrobial Resistance Profiles, and Resistance Genes with Human Extraintestinal Pathogenic Escherichia coli .Foodborne Pathogens and Disease , 16, 813-822. https://doi.org/10.1089/fpd.2019.2680

Carattoli, A., Zankari, E., García-Fernández, A., Voldby Larsen, M., Lund, O., Villa, L., ... Hasman, H. (2014). In silico detection and typing of plasmids using PlasmidFinder and plasmid multilocus sequence typing. Antimicrobial agents and chemotherapy , 58, 3895-903. https://doi.org/10.1128/AAC.02412-14

Cassini, A., Högberg, L.D., Plachouras, D., Quattrocchi, A., Hoxha, A., Simonsen, G.S., ... Hopkins, S. (2019). Attributable deaths and disability-adjusted life-years caused by infections with antibiotic-resistant bacteria in the EU and the European Economic Area in 2015: a population-level modelling analysis. The Lancet Infectious Diseases , 19, 56-66. https://doi.org/10.1016/S1473-3099(18)30605-4

Centers for Disease Control. (2019). Antibiotic Resistance Threats in the United States, 2019 . Retrieved from www.cdc.gov/DrugResistance/Biggest-Threats.html.

Cergole-Novella, M. C., Pignatari, A.C.C., \& Guth, B.E.C. (2015). Adhesion, biofilm and genotypic characteristics of antimicrobial resistant Escherichia coli isolates. Brazilian Journal of Microbiology , 46, 167-171. https://doi.org/10.1590/S1517-838246120140077

Cergole-Novella, M. C., Guth, B.E.C., Castanheira, M., Carmo, M.S., \& Pignatari, A.C.C. (2010). First description of bla CTX-M-14-andbla CTX-M-15- producing Escherichia coli isolates in Brazil. Microbial Drug Resistance , 16, 177-184. https://doi.org/10.1089/mdr.2010.0008.

CLSI. (2018). Performance Standards for Antimicrobial Disk and Dilution Susceptibility Tests for Bacteria Isolated From Animals (4th ed.). Wayne, PA: Clinical and Laboratory Standards Institute.

CLSI. (2020). Performance Standards for Antimicrobial Susceptibility Testing (30th ed.). Wayne, PA: Clinical and Laboratory Standards Institute.

Da Silveira, W.D., Ferreira, A., Lancellotti, M., Barbosa, I.A.G.C.D., Leite, D.S., De Castro, A.F.P., \& Brocchi, M. (2002). Clonal relationships among avian Escherichia coli isolates determined by enterobacterial repetitive intergenic consensus (ERIC)-PCR. Veterinary Microbiology , 89, 323-328. https://doi.org/10.1016/S0378-1135(02)00256-0

Dale, A.P. \& Woodford, N. (2015). Extra-intestinal pathogenicEscherichia coli (ExPEC): Disease, carriage and clones.Journal of Infection , 71, 615-626. https://doi.org/10.1016/j.jinf.2015.09.009

Dolejska, M. \& Literak, I. (2019). Wildlife is overlooked in the epidemiology of medically important antimicrobial resistant bacteria. Antimicrobial agents and chemotherapy , 63, 1-5. https://doi.org/10.1128/AAC.0116719

de Carvalho, M., Fernandes, M. R., Sellera, F. P., Lopes, R., Monte, D. F., Hippólito, A., ... Lincopan, N. (2020). International clones of extended-spectrum $\beta$-lactamase (CTX-M)-producing Escherichia coliin peri-urban wild animals, Brazil. Transboundary and emerging diseases , 00, 1-12. https://doi.org/10.1111/tbed.13558

EnteroBase. (2020). EnteroBase. Retrieved from http://enterobase.warwick.ac.uk/

Fuentes-Castillo, D., Farfán-López, M., Esposito, F., Moura, Q., Fernandes, M.R., Lopes, R., ... Lincopan, N. (2019). Wild owls colonized by international clones of extended-spectrum $\beta$-lactamase (CTX-M)-producing 
Escherichia coli and Salmonella Infantis in the Southern Cone of America. Science of the Total Environment , 674, 554-562. https://doi.org/10.1016/j.scitotenv.2019.04.149

Fuentes-Castillo, D., Esposito, F., Cardoso, B., Dalazen, G., Moura, Q., Fuga, ... Lincopan, N. (2020). Genomic Data reveal international lineages of critical priority Escherichia coli harbouring wide resistome in Andean condors (Vultur gryphus Linnaeus, 1758).Molecular ecology , 10.1111/mec.15455. https://doi.org/10.1111/mec.15455

Gonçalves, A., Igrejas, G., Radhouani, H., Estepa, V., Alcaide, E., Zorrilla, I., .. Poeta, P. (2012). Detection of extended-spectrum beta-lactamase-producing Escherichia coli isolates in faecal samples of Iberian lynx. Letters in Applied Microbiology , 54, 73-77. https://doi.org/10.1111/j.1472-765X.2011.03173.x

Gonçalves, A. C. M., Gabbay, Y. B., Mascarenhas, J. D. A., Yassaka, M. B., Moran, L. C., Fraga, V.D., ... Rossit, A. R. B. (2009). Short report: Calicivirus and Giardia lamblia are associated with diarrhea in human immunodeficiency virus-seropositive patients from southeast Brazil. American Journal of Tropical Medicine and Hygiene , 81, 463-466. https://doi.org/10.4269/ajtmh.2009.81.463

Gu, D., Dong, N., Zheng, Z., Lin, D., Huang, M., Wang, L., ... Chen, S. (2018). A fatal outbreak of ST11 carbapenem-resistant hypervirulent Klebsiella pneumoniae in a Chinese hospital: a molecular epidemiological study. The Lancet Infectious Diseases , 18, 37-46.https://doi.org/10.1016/S1473-3099(17)30489-9

Hernando-Amado, S., Coque, T. M., Baquero, F., \& Martínez, J. L. (2019). Defining and combating antibiotic resistance from One Health and Global Health perspectives. Nature Microbiology , 4,1432-1442. https://doi.org/10.1038/s41564-019-0503-9

Jarlier, V., Nicolas, M. H., Fournier, G., \& Philippon, A. (1988). Extended broad-spectrum beta-lactamases conferring transferable resistance to newer beta-lactam agents in Enterobacteriaceae: hospital prevalence and susceptibility patterns. Reviews of infectious diseases , 10, 867-78. https://doi.org/10.1093/clinids/10.4.867

Jenkins, C. (2015). Whole-Genome Sequencing Data for SerotypingEscherichia coli -It's Time for a Change! Journal of clinical microbiology , 53, 2402-3. https://doi.org/10.1128/JCM.01448-15

Lai, X. H., Arencibia, I., Johansson, A., Wai, S. N., Oscarsson, J., Kalfas, S., .. Uhlin, B. E. (2000). Cytocidal and apoptotic effects of the ClyA protein from Escherichia coli on primary and cultured monocytes and macrophages. Infection and immunity , 68, 4363-4367. https://doi.org/10.1128/IAI.68.7.4363-4367.2000

Lamas, I. R. \& Lins, L. V. (2009). Brazilian Merganser (Mergus octosetaceus ). In T.S. Schulenberg (Ed.), Neotropical Birds Online (Version1.0.). New York, USA: Cornell Laboratory of Ornithology. Retrieved from https://neotropical.birds.cornell.edu/Species-Account/nb/species/bramer1/overview

Lange, A., Schäfer, A., Bender, A., Steimle, A., Beier, S., Parusel, R., \& Frick, J. S. (2018). Galleria mellonella : A novel invertebrate model to distinguish intestinal symbionts from pathobionts. Frontiers in Immunology , 9, 1-12. https://doi.org/10.3389/fimmu.2018.02114

Larsen, M. V., Cosentino, S., Rasmussen, S., Friis, C., Hasman, H., Marvig, R.L., ... Lund, O. (2012). Multilocus sequence typing of total-genome-sequenced bacteria. Journal of clinical microbiology , 50, 135561. https://doi.org/10.1128/JCM.06094-11

Larsson, D. G. J., Andremont, A., Bengtsson-Palme, J., Brandt, K. K., de Roda-Husman, A. M., Fagerstedt, P., ... Wernersson, A.-S. (2018). Critical knowledge gaps and research needs related to the environmental dimensions of antibiotic resistance. Environment International , 117, 132-138. https://doi.org/10.1016/j.envint.2018.04.041

Lithgow, J. K., Haider, F., Roberts, I. S., \& Green, J. (2007). Alternate SlyA and H-NS nucleoprotein complexes control hlyE expression in Escherichia coli K-12. Molecular microbiology , 66, 685-698. https://doi.org/10.1111/j.1365-2958.2007.05950.x 
McKinnon, J., Chowdhury, P. R., \& Djordjevic, S. P. (2018). Genomic analysis of multidrug-resistant Escherichia coli ST58 causing urosepsis. International Journal of Antimicrobial Agents , 52, 430-435. https://dx.doi.org/10.1016/j.ijantimicag.2018.06.017

Oscarsson, J., Mizunoe, Y., Li, L., Lai, X. H., Wieslander, A., \& Uhlin, B. E. (1999). Molecular analysis of the cytolytic protein ClyA (SheA) from Escherichia coli . Molecular microbiology , 32, 1226-1238. https://doi.org/10.1046/j.1365-2958.1999.01435.x

Osek, J., Weiner, M., \& Hartland, E. L. (2003). Prevalence of the lpfO113 gene cluster among Escherichia coli O157 isolates from different sources. Veterinary Microbiology , 96, 259-266. https://doi.org/10.1016/j.vetmic.2003.07.002

Pettersen, V. K., Mosevoll, K. A., Lindemann, P. C., \& Wiker, H. G. (2016). Coordination of metabolism and virulence factors expression of extraintestinal pathogenic Escherichia coli purified from blood cultures of patients with sepsis. Molecular and Cellular Proteomics , 15, 2890-2907. https://dx.doi.org/10.1074\%2Fmcp.M116.060582

Ramey, A. M. \& Ahlstrom, C. A. (2020). Antibiotic resistant bacteria in wildlife: perspectives on trends, acquisition and dissemination, data gaps, and future directions. Journal of Wildlife Diseases , 56, 1-15. https://doi.org/10.7589/2019-04-099

Robinson, A. E., Heffernan, J. R., \& Henderson, J. P. (2018). The iron hand of uropathogenic Escherichia coli : The role of transition metal control in virulence. Future Microbiology , 13, 813-829. https://doi.org/10.2217/fmb-2017-0295

Ruiz-Castellano, C., Tomás, G., Ruiz-Rodríguez, M., Martín-Gálvez, D., \& Soler, J. J. (2016). Nest Material Shapes Eggs Bacterial Environment.PLoS ONE ,11, 1-21. https://doi.org/10.1371/journal.pone.0148894

Sacramento, A. G., Fernandes, M. R., Sellera, F. P., Muñoz, M. E., Vivas, R., Dolabella, S. S., \& Lincopan, N. (2018). Genomic analysis of MCR-1 and CTX-M-8 co-producing Escherichia coli ST58 isolated from a polluted mangrove ecosystem in Brazil. Journal of Global Antimicrobial Resistance , 15, 288-289. https://doi.org/10.1016/j.jgar.2018.10.024

Sacristán, I., Esperón, F., Acuña, F., Aguilar, E., García, S., López, M. J., .. Millán, J. (2020). Antibiotic resistance genes as landscape anthropization indicators: Using a wild felid as sentinel in Chile. Science of the Total Environment , 703, 134900. https://doi.org/10.1016/j.scitotenv.2019.134900

Santos, A. C. M., Zidko, A. C. M., Pignatari, A. C., \& Silva, R. M. (2013). Assessing the diversity of the virulence potential ofEscherichia coli isolated from bacteremia in São Paulo, Brazil.Brazilian Journal of Medical and Biological Research , 46, 968-973. https://doi.org/10.1590/1414-431X20133184

Sarowska, J., Futoma-Koloch, B., Jama-Kmiecik, A., Frej-Madrzak, M., Ksiazczyk, M., Bugla-Ploskonska, G., \& Choroszy-Krol, I. (2019). Virulence factors, prevalence and potential transmission of extraintestinal pathogenic Escherichia coli isolated from different sources: Recent reports. Gut Pathogens, 11, 1-16. https://doi.org/10.1186/s13099-019-0290-0

Sevilla, E., Marín, C., Delgado-Blas, J. F., Gonzalez-Zorn, B., Vega, S., Kuijper, E., .. Mainar-Jaime, R. C. (2020). Wild griffon vultures (Gyps fulvus ) fed at supplementary feeding stations: Potential carriers of pig pathogens and pig-derived antimicrobial resistance? Transboundary and Emerging Diseases , 00, 1-11. https://doi.org/10.1111/tbed.13470

Torres, A. G. (2016). Escherichia coli in the Americas . Switzerland: Springer International Publishing.

Tsai, C. J. Y., Loh, J. M. S., \& Proft, T. (2016). Galleria mellonella infection models for the study of bacterial diseases and for antimicrobial drug testing. Virulence , 7, 214-229. https://doi.org/10.1080/21505594.2015.1135289 
Vittecoq, M., Godreuil, S., Prugnolle, F., Durand, P., Brazier, L., Renaud, N., ... Renaud, F. (2016). Antimicrobial resistance in wildlife. Journal of Applied Ecology , 53, 519-529. https://doi.org/10.1111/13652664.12596

White, A. \& Hughes, J. M. (2019). Critical Importance of a One Health Approach to Antimicrobial Resistance. EcoHealth , 16, 404-409. https://doi.org/10.1007/s10393-019-01415-5

Wyborn, N. R., Clark, A., Roberts, R. E., Jamieson, S. J., Tzokov, S., Bullough, P. A., .. \& Green, J. (2004). Properties of haemolysin E (HlyE) from a pathogenic Escherichia coli avian isolate and studies of HlyE export. Microbiology , 150, 1495-1505. https://doi.org/10.1099/mic.0.26877-0

Zankari, E., Hasman, H., Cosentino, S., Vestergaard, M., Rasmussen, S., Lund, O., .. Larsen, M.V. (2012). Identification of acquired antimicrobial resistance genes. The Journal of antimicrobial chemotherapy , 67, 2640-4. https://doi.org/10.1093/jac/dks261

Zurfluh, K., Albini, S., Mattmann, P., Kindle, P., Nuesch-Inderbinen, M., Stephan, R., \&Vogler, B. R. (2019). Antimicrobial resistant and extended-spectrum $\beta$-lactamase producing Escherichia coli in common wild bird species in Switzerland. MicrobiologyOpen , 8, e845. https://doi.org/10.1002/mbo3.845

Table 1. Virulome of MDR CTX-M-8-producing Escherichia coli PMPU strain isolated from hemorrhagic pulmonary tissue of an 8-day-old Brazilian Merganser.

\begin{tabular}{ll}
\hline Characteristics & Virulence genes \\
Adherence & fimBCEFGHI, cfaABCD, lpfAO113, matF, stgBCD, ycbFRSTUV \\
Fimbriae & flgABCDEFGHIJKLN, flhABCDE, fliADEFGHIJKLMNOPQRSTYZ, flk \\
Flagella & hofCB \\
Pilus & ppdABCD, hofQ, ygdB, yggR, b2854, b2972 \\
Adherence hemorrhagic coli pilus & eaeH, ecpRABCD, ehaABG \\
Adhesins & csgBCDEFG \\
Curli fibers & cib \\
Protectins and invasins & iss \\
Colicin & ibeBC \\
Increased serum survival & \\
Invasin & entBCEFS, fes \\
Iron acquisition systems & epABCD \\
Enterobactin & \\
Ferrienterobactin & hlyE \\
Toxins & \\
Hemolysin E & gspCDEFGHIJKLM, ygh $G$ \\
Secretion systems components & espL3-4, espR1, espX1-5, eprHIJK \\
Type II secretion system & \\
Type III secretion system & gadX \\
Others & cadA \\
Glutamate decarboxylase & cheABRMWYZ, motAB \\
Lysine decarboxylase & epaOPQRS \\
Chemotaxis &
\end{tabular}

\section{FIGURE LEGENDS}

Figure 1. Microscopic findings in an 8-day-old Brazilian merganser (Mergus octosetaceus ) with colibacillosis. In a: Lungs, note congestion of alveolar capillaries and perivascular edema (Black arrow). In b: Liver, note hepatocellular swelling and intracytoplasmic vacuolation (Black arrow). Hematoxylin and eosin staining. 
Figure 2. Resistome of multidrug-resistant CTX-M-8-producingEscherichia coli PMPU strain. Columns show genes encoding resistance to antibiotics, disinfectants, heavy metals and acid or basic environment.

${ }^{a}$ Mutations in quinolone resistance-determining region (QRDR).

Figure 3. Phylogeny of CTX-M-8-producing Escherichia coli isolate from a Brazilian merganser (Mergus octosetaceus ), in relation to an international E. coli collection. The image shows a minimum spanning tree based on wgMLST of 123 worldwide distributed $E$. coli strains belonging to ST58, constructed by the MSTree V2 tool from EnteroBase. The figure was generated with iTOL v.5.5 (https://itol.embl.de). Interactive versions of the tree can be found at https://itol.embl.de/tree/20014463144294501588789515. Colored circles represent sources of origin. Each isolate is indicated by the country of origin.
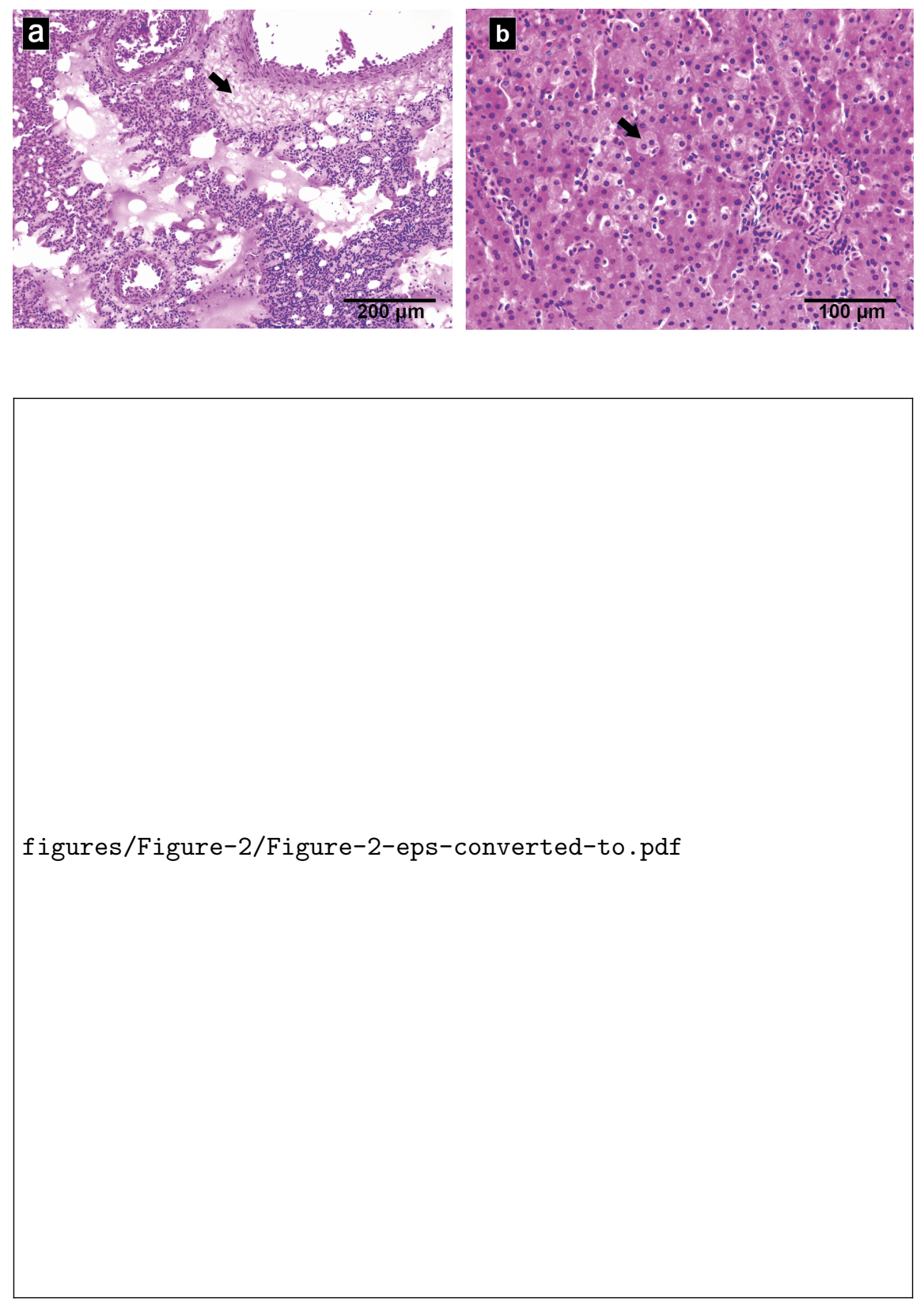
figures/Figure-3/Figure-3-eps-converted-to.pdf 